\title{
Gas generation and overpressure: Effects on seismic attributes
}

\author{
José M. Carcione* and Anthony F. Gangi ${ }^{\ddagger}$
}

\begin{abstract}
Drilling of deep gas resources is hampered by high risk associated with unexpected overpressure zones. Knowledge of pore pressure using seismic data, as for instance from seismic-while-drilling techniques, will help producers plan the drilling process in real time to control potentially dangerous abnormal pressures.

We assume a simple basin-evolution model with a constant sedimentation rate and a constant geothermal gradient. Oil/gas conversion starts at a given depth in a reservoir volume sealed with faults whose permeability is sufficiently low so that the increase in pressure caused by gas generation greatly exceeds the dissipation of pressure by flow. Assuming a first-order kinetic reaction, with a reaction rate satisfying the Arrhenius equation, the oil/gas conversion fraction is calculated. Balancing mass and volume fractions in the pore space yields the ex-
\end{abstract}

cess pore pressure and the fluid saturations. This excess pore pressure determines the effective pressure, which in turn determines the skeleton bulk moduli. If the generated gas goes into solution in the oil, this effect does not greatly change the depth and oil/gas conversion fraction for which the hydrostatic pressure approaches the lithostatic pressure.

The seismic velocities versus pore pressure and differential pressure are computed by using a model for wave propagation in a porous medium saturated with oil and gas. Moreover, the velocities and attenuation factors versus frequency are obtained by including rock-frame/fluid viscoelastic effects to match ultrasonic experimental velocities. For the basin-evolution model used here, pore pressure is seismically visible when the effective pressure is less than about $15 \mathrm{MPa}$ and the oil/gas conversion is about $2.5 \%$ percent.

\section{INTRODUCTION}

In deeply buried oil reservoirs, oil-to-gas cracking may increase the pore pressure to reach or exceed the lithostatic pressure (Chaney, 1950; Barker, 1990; Luo and Vasseur, 1996). Oil can be generated from kerogen-rich source rocks and can flow through a carrier bed to a sandstone reservoir rock. Excess pore-fluid pressures in sandstone reservoirs are generated when the rate of volume created by the transformation of oil to gas is more rapid than the rate of volume loss by fluid flow. If the reservoir is sealed on all sides by an impermeable shale or limestone, then the condition of a closed system will be satisfied for gas generation. Because of the presence of semivertical fault planes and compartmentalization, this condition holds for most North Sea reservoirs. Moreover, gas generation significantly decreases the relative permeability (Luo and Vasseur, 1996).

Berg and Gangi (1999) developed a simple model to calculate the excess pore pressure as a function of the fraction of kerogen converted to oil and the fraction of oil converted to gas. The oil/gas conversion ratio is computed as a function of time, for a given sedimentation rate and geothermal gradient. Then Berg and Gangi (1999) determined the excess pore pressure resulting from the oil/gas conversion with burial time, which is derived by balancing mass and volume changes in the pore space. Carcione (2000) applied this model of the calculation of the kerogen/oil conversion to obtain the seismic properties of overpressured shales. Here, we use an extended version of this model for computing the porosity variations and fluid saturations as a function of the excess pore pressure. We consider two cases, when the generated gas goes into solution in the oil and when it remains as free gas. When gas goes into solution in dead oils, it generates live oils. The analysis is based on the equations for live oil given by Batzle and Wang (1992). The solubilities of hydrocarbon gases in water are very small compared with the solubilities of the same gases in oil.

Seismic velocities have been used to predict pore pressure, with most of the models based on empirical relations (Dutta and Levin, 1990). Attenuation has been studied mainly at ultrasonic frequencies, and a few works have focused on vertical seismic profiling (VSP) data and sonic data (Hague, 1981;

Manuscript received by the Editor September 3, 1998; revised manuscript received October 25, 1999.

*Osservatorio Geofisico Sperimentale, P.O. Box 2011, 34016 Trieste, Italy. E-mail: jcarcione@ogs.trieste.it.

†Department of Geology and Geophysics, Texas A\&M University, College Station, Texas 77843-3114. E-mail: gangi@tamu.edu.

(C) 2000 Society of Exploration Geophysicists. All rights reserved. 
Dasios et al., 1998a). Recently, attenuation obtained from surface seismic data has been used successfully to predict lithology and pore pressure (Helle et al., 1993; Dasios et al., 1998b). In this work, the seismic properties are calculated by using a modification of Biot's theory for fully saturated porous solids. The effective parameters for the fluid mixture are computed by the average formulas given by Berryman et al. (1988).

The wave velocities of the dry rock, determined by laboratory measurements, give estimates of its pore compressibility and bulk moduli in the low-frequency (relaxed) regime. Because dissipation mechanisms are caused mainly by grain/fluid interactions, dry-rock velocities are frequency independent. On the other hand, measurements of ultrasonic saturated-rock velocities provide the high-frequency-limit (unrelaxed) velocities, which can be used to estimate the amount of velocity dispersion between the seismic and ultrasonic ranges and, therefore, the amount of attenuation. By using this information, the phase velocities and attenuation factors are computed as a function of frequency.

\section{GAS GENERATION AND ROCK PROPERTIES}

First, We will introduce some useful definitions about the different pressures considered in this work. Pore pressure, also known as formation pressure, is the in situ pressure of the fluids in the pores. The pore pressure is equal to the hydrostatic pressure when the pore fluids only support the weight of the overlying pore fluids (mainly brine). The lithostatic or confining pressure results from the weight of overlying sediments, including the pore fluids. In the absence of any state of stress in the rock, the pore pressure attains lithostatic pressure and the fluids support all the weight. However, fractures perpendicular to the minimum compressive stress direction appear for a given pore pressure, typically $70-90 \%$ of the confining pressure. In this case, the fluid escapes from the pores, and pore pressure decreases. A rock is said to be overpressured when its pore pressure is significantly greater than hydrostatic pressure. The difference between pore pressure and hydrostatic pressure is called differential pressure. Acoustic and transport properties of rocks generally depend on effective pressure, a combination of pore and confining pressures [see equation (20)]. Various physical processes cause anomalous pressures on an underground fluid. The most common causes of overpressure are compaction disequilibrium and cracking, i.e., oil-to-gas conversion (Mann and Mackenzie, 1990; Luo and Vasseur, 1996).

Let us assume a reservoir at depth $z$. The lithostatic pressure for an average sediment density of $\bar{\rho}$ is equal to $p_{c}=\bar{\rho} g z$, where $g$ is the acceleration of gravity. On the other hand, the hydrostatic pore pressure is approximately $p_{H}=\rho_{w} g z$, where $\rho_{w}$ is the density of water.

For a constant sediment burial rate, $S$, and a constant geothermal gradient, $G$, the temperature variation of a particular sediment volume is

$$
T=T_{0}+G z, \quad z=S t
$$

with a surface temperature $T_{0}$ at time $t=0$. Typical values of $G$ range from 20 to $30^{\circ} \mathrm{C} / \mathrm{km}$, and $S$ may range between 0.05 and $0.5 \mathrm{~km} / \mathrm{m} . \mathrm{y}$. (m.y. $=$ million years).

Assume that at time $t_{i}$, corresponding to depth $z_{i}$, the reservoir volume has been saturated with oil which flowed from an adjacent source rock, and that the volume is "closed." That is, the permeability of the sealing faults is sufficiently low so that the rate of pressure increase caused by gas generation greatly exceeds the dissipation of pressure by flow. Pore-pressure excess is intended to be above hydrostatic.

\section{Oil/gas generation rate}

The mass of convertible oil changes with time $t$ at a rate proportional to the mass present. Assuming a first-order kinetic reaction (Luo and Vasseur, 1996; Berg and Gangi, 1999),

$$
\frac{d M_{o}}{d t}=-r_{o}(t) M_{o}(t)
$$

or

$$
M_{o}(t)=M_{o i} \exp \left[-\int_{t_{i}}^{t} r_{o}(t) d t\right]
$$

where $r_{o}(t)$ is the reaction rate, $M_{o}(t)$ is the mass of convertible oil at time $t$, and $M_{o i}$ is the initial oil mass. The fraction of oil converted to gas is $F(t)=\left[M_{o i}-M_{o}(t)\right] / M_{o i}$ :

$$
F(t)=1-\exp \left[-\int_{t_{i}}^{t} r_{o}\left(t^{\prime}\right) d t^{\prime}\right] \equiv 1-\exp [-\Phi(t)] .
$$

The reaction rate follows the Arrhenius equation (Luo and Vasseur, 1996)

$$
r_{o}(t)=A \exp [-E / R T(t)]
$$

where $E$ is the oil/gas activation energy, $R=1.986 \mathrm{cal} / \mathrm{mol}{ }^{\circ} \mathrm{K}$ is the gas constant, $A$ is the oil/gas reaction rate at infinite temperature, and $T(t)$ is the absolute temperature in ${ }^{\circ} \mathrm{K}$ given by

$$
T=T_{0}+H t, \quad H=G S .
$$

With this temperature dependence, the integral $\Phi(t)$ becomes

$$
\begin{aligned}
\Phi(t) & =\int_{t_{i}}^{t} r_{o}\left(t^{\prime}\right) d t^{\prime}=\frac{A}{H} \int_{T_{i}}^{T} \exp \left(-E / R T^{\prime}\right) d T^{\prime}, \\
T_{i} & =T_{0}+H t_{i}
\end{aligned}
$$

or

$$
\begin{aligned}
\Phi(t)= & \frac{A}{H}\left[T \int_{1}^{\infty} \exp (-E x / R T) \frac{d x}{x^{2}}\right. \\
& \left.-T_{i} \int_{1}^{\infty} \exp \left(-E x / R T_{i}\right) \frac{d x}{x^{2}}\right]
\end{aligned}
$$

For values of $E / R T$ greater than 10 , the exponential integral can be approximated by (Gautschi and Cahill, 1964, 248, Table 5.5)

$$
\int_{1}^{\infty} \exp (-E x / R T) \frac{d x}{x^{2}} \cong \frac{\exp (-E / R T)}{2+E / R T},
$$


with an error of $1.3 \%$ or less. Then the integral $\Phi$ becomes

$$
\Phi(T(t))=\frac{A}{H}\left[\frac{T \exp (-E / R T)}{2+E / R T}-\frac{T_{i} \exp \left(-E / R T_{i}\right)}{2+E / R T_{i}}\right] .
$$

The activation energy and infinite-temperature rate used in this work are $E=52 \mathrm{kcal} / \mathrm{mol}$ and $A=5.5 \times 10^{26} / \mathrm{m}$.y. (Luo and Vasseur, 1996; Berg and Gangi, 1999).

\section{Oil/gas conversion factor and excess pore pressure}

We are interested primarily in a first-order solution which shows the large effect of oil-to-gas conversion on seismic properties. In this sense, following Berg and Gangi (1999), we assume:

1) The compressibilities of oil and water are independent of pressure and temperature, and that of the rock is independent of temperature but depends on pressure. That this is the case can be seen from the results given by Batzle and Wang (1992) in their Figures 5 and 13, in which they show that the density is almost a linear function of temperature and pressure. This means that the mentioned properties are approximately constant (see also their Figure 7, in which the oil compressibility remains almost constant when going from low temperature and low pressure to high temperature and high pressure).

2) The gas satisfies the van der Waals equation (Friedman, 1963):

$$
\left(p+a \rho_{g}^{2}\right)\left(1-b \rho_{g}\right)=\rho_{g} R T,
$$

where $p$ is the gas pressure, $\rho_{g}$ is the gas density, $T$ is the absolute temperature, and $R$ is the gas constant. Moreover, $a=0.225 \mathrm{~Pa}\left(\mathrm{~m}^{3} / \mathrm{mole}\right)^{2}=879.9 \mathrm{MPa}\left(\mathrm{cm}^{3} / \mathrm{g}\right)^{2}$, and $b=4.28 \times 10^{-5} \mathrm{~m}^{3} / \mathrm{mole}=2.675 \mathrm{~cm}^{3} / \mathrm{g}$ (one mole of methane, $\mathrm{CH}_{4}$, corresponds to $16 \mathrm{~g}$ ). The van der Waals equation is a good approximation of the behavior of natural gas, as shown by Berg and Gangi (1999), where the differences between the experimental data-as represented by Standing's results (Standing, 1952) —and the van der Waals results are only about $15 \%$ over the depths of interest.

3) The initial pore fluids are water and oil.

The excess pore pressure at depth $z$ is $p-p_{H}$, where $p_{H}$ is the hydrostatic pore pressure at depth $z$, and $p$ is the pore pressure when a fraction $F$ of oil has been converted to gas $(F=0$ and $p=p_{i}=p_{H}$ at time $\left.t_{i}\right)$. The mass balance is

$$
\rho_{g} V_{g}=F \rho_{o i} V_{o i}
$$

where $\rho_{o i}$ is the initial oil density, $V_{g}$ is the converted gas volume, and $V_{o i}$ is the initial oil volume. Assumption (3) implies

$$
V_{p i}=V_{w i}+V_{o i} \quad \text { or } \quad V_{p i} / V_{w i}=1+v,
$$

where $V_{p i}$ is the initial pore volume and $v=V_{o i} / V_{w i}$.

The compressibilities of the oil, water, and pore space are defined, respectively, as

$$
c_{o}=-\frac{1}{V_{o}} \frac{d V_{o}}{d p}, \quad c_{w}=-\frac{1}{V_{w}} \frac{d V_{w}}{d p}, \quad c_{p}=-\frac{1}{V_{p}} \frac{d V_{p}}{d p_{e}},
$$

where $p_{e}$ is the effective pressure and $c_{p}$ can be obtained from the compressibility at zero pore pressure.

In general, compressional and shear-wave velocities depend on effective pressure $p_{e}=p_{c}-n p$, where $n \leq 1$ is the effective stress coefficient. Note that the effective pressure equals the confining pressure at zero pore pressure. It is found that $n \approx 1$ for static measurements of the compressibilities (Zimmerman et al., 1986), while $n$ is approximately linearly dependent on the differential pressure $p_{d}=p_{c}-p$ in dynamic experiments (Gangi and Carlson, 1996; Prasad and Manghnani, 1997):

$$
n=n_{0}-n_{1} p_{d}
$$

where $n_{0}$ and $n_{1}$ are constant coefficients.

We assume the following functional form for $c_{p}$ as a function of effective pressure:

$$
c_{p}=c_{p}^{\infty}+\beta \exp \left(-p_{e} / p^{*}\right)
$$

where $c_{p}^{\infty}, \beta$, and $p^{*}$ are coefficients obtained by fitting the experimental data. On the other hand, the thermal expansion coefficients for oil, water, and pore space are defined, respectively, as

$$
\alpha_{o}=\frac{1}{V_{o}} \frac{d V_{o}}{d T}, \quad \alpha_{w}=\frac{1}{V_{w}} \frac{d V_{w}}{d T}, \quad \alpha_{p}=\frac{1}{V_{p}} \frac{d V_{p}}{d T} .
$$

Integration from $p_{i}\left(p_{e i}\right)$ to $p\left(p_{e}\right)$ and from $T_{i}$ to $T_{i}+\Delta T$, where $\Delta T=T-T_{i}$, yields

$$
\begin{aligned}
V_{o}(p, T) & =V_{o i}\left[\exp \left(-c_{o} \Delta p+\alpha_{o} \Delta T\right)\right], \\
V_{w}(p, T) & =V_{w i}\left[\exp \left(-c_{w} \Delta p+\alpha_{w} \Delta T\right)\right],
\end{aligned}
$$

and

$$
V_{p}(p, T)=V_{p i}\left\{\exp \left[E(\Delta p)+\alpha_{p} \Delta T\right]\right\}
$$

where

$$
E(\Delta p)=-c_{p}^{\infty} \Delta p_{e}+\beta p^{*}\left[\exp \left(-p_{e} / p^{*}\right)-\exp \left(-p_{e i} / p^{*}\right)\right]
$$

and $\Delta p=p-p_{i}=p-p_{H i}$. Using equation (15), we can write the effective pressure as

$$
p_{e}=p_{c}-\left(n_{0}-n_{1} p_{c}\right) p-n_{1} p^{2}
$$

Berg and Gangi (1999) assume a constant pore compressibility $c_{p}$. In this case, $E(\Delta p)=-c_{p} \Delta p$.

In principle, if no gas goes into solution in the oil, the pore volume at pore pressure $p$ and temperature $T$ is given by

$$
\begin{aligned}
V_{p}(p, T)= & V_{p i}\left\{\exp \left[E(\Delta p)+\alpha_{p} \Delta T\right]\right\} \\
= & V_{w i}\left[\exp \left(-c_{w} \Delta p+\alpha_{w} \Delta T\right)\right]+(1-F) V_{o i} \\
& \times\left[\exp \left(-c_{o} \Delta p+\alpha_{o} \Delta T\right)\right]+V_{g}(p, T)
\end{aligned}
$$

since $(1-F)$ of the initial oil remains at pressure $p$. Substituting equation (12) into equation (21) and dividing through by $V_{w i}$ yields 


$$
\rho_{o}=\frac{\rho_{0}+\left(0.00277 p-1.71 \times 10^{-7} p^{3}\right)\left(\rho_{0}-1.15\right)^{2}+3.49 \times 10^{-4} p}{0.972+3.81 \times 10^{-4}(T+17.78)^{1.175}} .
$$

$$
\begin{aligned}
(1 & \left.+\frac{1}{v}\right)\left\{\exp \left[E(\Delta p)+\alpha_{p} \Delta T\right]\right\} \\
= & \frac{1}{v} \exp \left(-c_{w} \Delta p+\alpha_{w} \Delta T\right) \\
& +(1-F)\left[\exp \left(-c_{o} \Delta p+\alpha_{o} \Delta T\right)\right]+F \rho_{o i} / \rho_{g} .
\end{aligned}
$$

In the absence of water, $1 / v \rightarrow 0$.

There are two possibilities when the generated gas goes into solution: (1) all the gas dissolves in the oil, which occurs at the beginning of the conversion process down to a critical depth, and (2) a fraction of gas goes into solution in the oil and the rest remains as free gas. The process can be approximated, as illustrated in Figure 1: It begins at stage (1); in stage (2), a volume $V_{g}=V_{g 1}+V_{g 2}$ of gas is generated, with $V_{g 1}$ going immediately into solution and $V_{g 2}$ remaining as free gas (stage 3 ). In the following, we obtain the balance equations, equivalent to equation (22) and the fractions of solid, water, live oil, and free gas at stage (3), for the two cases described above.

The volume ratio of liberated gas to remaining oil at atmospheric pressure and $15.6^{\circ} \mathrm{C}$ is

$$
R_{G}=0.02123 G_{r}\left[p \exp \left(\frac{4.072}{\rho_{0}}-0.00377 T\right)\right]^{1.205},
$$

where $G_{r}$ is the gas gravity and $\rho_{0}$ is the oil density at atmospheric pressure and temperature (Batzle and Wang, 1992); $R_{G}$, given in liters of gas/liters of oil, represents the maximum amount of gas that can be dissolved in the oil. At depth $z$, temperature $T$, and pressure $p$, the equivalent ratio is

$$
R_{G}^{\prime}=\frac{\rho_{g s}}{\rho_{g}} \frac{\rho_{o}}{\rho_{o s}} R_{G},
$$

where $\rho_{g s}, \rho_{o s}$ and $\rho_{g}, \rho_{o}$ are the gas and oil densities at the surface and at depth $z$, respectively. The gas densities can be computed from the van der Waals equation (11), and the oil density is given by

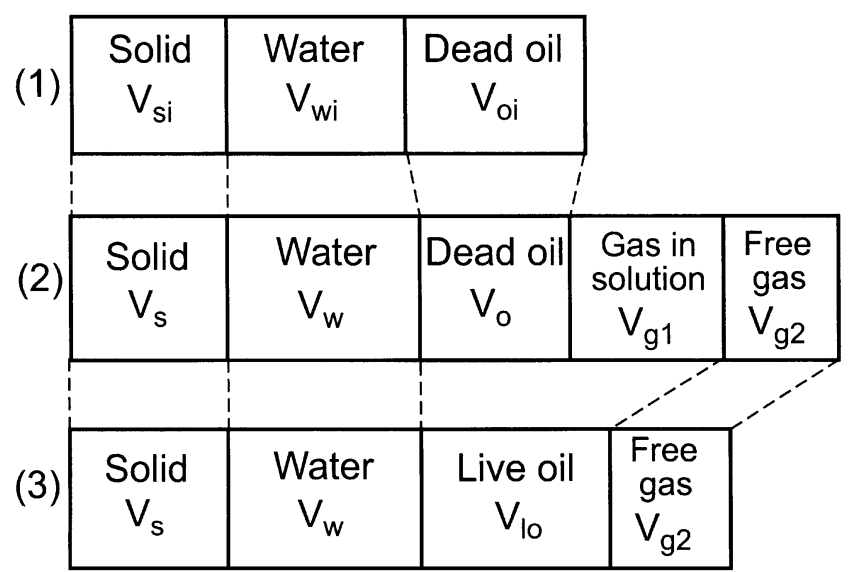

FIG. 1. Oil-to-gas conversion process. Part of the generated gas goes into solution.
Oil densities are given in $\mathrm{g} / \mathrm{cm}^{3}$, temperature $T$ in ${ }^{\circ} \mathrm{C}$, and pressure $p$ in MPa. Because $R_{G}^{\prime}$ is the maximum volume of gas $\left(V_{g 1}\right)$ that can be dissolved in the remaining volume of dead oil, we have

$$
R_{G}^{\prime}=\left[\frac{\rho_{o}}{(1-F) \rho_{o i}}\right] \frac{V_{g 1}}{V_{o i}} .
$$

The volume of free gas $\left(V_{g 2}\right)$ is equal to the total volume of generated gas $\left(V_{g}\right)$ minus the maximum volume of dissolved gas $\left(V_{g 1}\right)$,

$$
\begin{aligned}
V_{g 2} & =F \frac{\rho_{o i}}{\rho_{g}} V_{o i}-(1-F) \frac{\rho_{o i}}{\rho_{o}} R_{G}^{\prime} V_{o i} \\
& =\left\{\left[\frac{F}{\rho_{g}}-\frac{(1-F)}{\rho_{o}} R_{G}^{\prime}\right] \rho_{o i}\right\} V_{o i} \equiv \delta V_{o i},
\end{aligned}
$$

where equation (26) has been used. There is a critical depth $z_{c}$ for which the gas saturates the oil. Above this depth (which corresponds to a critical pressure $p_{c}$ ), all the gas goes into solution in the oil (note that the process is a dynamic one, in which gas is generated continuously). This occurs when the volume of free gas [equation (27)] is equal to zero, i.e., when a fraction

$$
F_{c}=\frac{\rho_{g} R_{G}^{\prime}}{\rho_{o}+\rho_{g} R_{G}^{\prime}}
$$

of oil has been converted to gas, at the critical pressure $p_{c}$. Below the critical depth, the pore space will contain live oil mixed with free gas. To compute $z_{c}$ and $F_{c}$, we assume that the system is subject to the pore-pressure profile obtained from equation (22), which is the situation shown in stage (2) of Figure 1 , before the gas goes into solution. Then $z_{c}$ is the depth for which $\delta=0$ [see equation (27)] or $F=F_{c}$. It can be argued that after the gas goes into solution, the pressure will decrease. However, if this occurs, part of the gas will go out of solution immediatley (as free gas) and the pressure will increase again to the previous value. It can be shown that by choosing, for instance, the hydrostatic pressure $p_{H}$ as the reference pressure profile, instead of that obtained from equation (22), the critical depth will be different, but the results will not be affected greatly.

The density of the live oil at and below the critical depth is given by

$$
\begin{aligned}
\rho_{l o}= & \rho_{G}+\left(0.00277 p-1.71 \times 10^{-7} p^{3}\right)\left(\rho_{G}-1.15\right)^{2} \\
& +3.49 \times 10^{-4} p
\end{aligned}
$$

with

$$
\rho_{G}=\left(\rho_{0}+0.0012 G_{r} R_{G}\right) / B_{0},
$$

the saturation density, and

$$
B_{0}=0.972+0.00038\left[2.4 R_{G}\left(\frac{G_{r}}{\rho_{0}}\right)^{1 / 2}+T+17.8\right]^{1.175},
$$


the oil-volume factor (Batzle and Wang, 1992). When computing the density of live oil, the temperature effect was considered twice in Batzle and Wang (1982), in $B_{0}$ and in their equation (19) (M. Batzle, personal communication). Equation (29) gives the correct density. At the initial depth $z_{i}$ there is no generated gas, and the oil density is that of the dead oil $\rho_{o i}$.

When $z_{i} \leq z \leq z_{c}$ [case (1)], all the gas goes into solution, but the oil is not saturated because it absorbs less than $R_{G}^{\prime}$ liters of gas; it absorbs $\left(F / F_{c}\right) R_{G}^{\prime}$. The density, $\rho_{l o}$, of live oil is obtained by substituting $R_{G}$ by $\left(F / F_{c}\right) R_{G}$ in equations (29), (30), and (31). Because, in this range, the mass of live oil is equal to the initial mass of dead oil, the volume of live oil is

$$
V_{l o}=\frac{\rho_{o i}}{\rho_{l o}} V_{o i} \equiv \eta V_{o i}
$$

Then the balance equation above the critical depth is

$$
\begin{aligned}
& \left(1+\frac{1}{v}\right)\left\{\exp \left[E(\Delta p)+\alpha_{p} \Delta T\right]\right\} \\
& =\frac{1}{v} \exp \left(-c_{w} \Delta p+\alpha_{w} \Delta T\right)+\eta
\end{aligned}
$$

We now consider case (2), i.e., when $z>z_{c}$ and the pore space is filled with live oil and free gas. The mass of live oil $\rho_{l o} V_{l o}$ is equal to the mass of remaining dead oil $(1-F) \rho_{o i} V_{o i}$ plus the mass of gas going into solution. The latter is equal to the maximum volume of gas $V_{g 1}$ that can be dissolved in the remaining dead oil [equation (26)], multiplied by the gas density $\rho_{g}$. Then the volume of live oil is

$$
V_{l o}=\left[\frac{\rho_{o i}}{\rho_{l o}}(1-F)\left(1+R_{G}^{\prime} \frac{\rho_{g}}{\rho_{o}}\right)\right] V_{o i} \equiv \gamma V_{o i} .
$$

Below the critical depth, the density of live oil, $\rho_{l o}$, is obtained from equation (29). Taking into account that the volume of free gas is given by equation (27) and using (34), the balance equation is

$$
\begin{gathered}
\left(1+\frac{1}{v}\right)\left\{\exp \left[E(\Delta p)+\alpha_{p} \Delta T\right]\right\}=\frac{1}{v} \exp \left(-c_{w} \Delta p\right. \\
\left.+\alpha_{w} \Delta T\right)+\gamma+F \rho_{o i} / \rho_{g}-R_{G}^{\prime}(1-F) \rho_{o i} / \rho_{o}
\end{gathered}
$$

Equations (33) and (35) relate the pore pressure $p$ to the converted oil/gas fraction $F$ at the confining pressure $p_{c}$ and temperature $T$. All these quantities depend on the deposition time $t$. Note also that $\eta, \rho_{g}, \gamma$, and $R_{G}^{\prime}$ depend on pore pressure and temperature.

\section{Volume fractions and saturations}

The fractions of live oil and water at stage (3), above the critical depth $z_{c}$ (see Figure 1), are equal to the corresponding volumes divided by the total volume $V_{s}+V_{w}+V_{l o}$. The volume of the solid part can be obtained from the initial porosity $\phi_{i}$ and the initial pore volume $V_{p i}$, because $\phi_{i}=V_{p i} /\left(V_{p i}+V_{s}\right)$; thus, $V_{s}=V_{p i}\left(1 / \phi_{i}-1\right)$, and by using $(13), V_{s} / V_{o i}=(1+1 / v)$ $\left(1 / \phi_{i}-1\right)$. It can be shown easily that the fractions of live oil, water, and solid are given by

$$
\begin{aligned}
\phi_{l o} & =\eta\left[\left(V_{s} / V_{o i}\right)+\left(V_{w} / v V_{w i}\right)+\eta\right]^{-1}, \\
\phi_{w} & =\frac{1}{v \eta}\left(V_{w} / V_{w i}\right) \phi_{l o},
\end{aligned}
$$

and

$$
\phi_{s}=1-\phi_{l o}-\phi_{w}
$$

respectively, where the volume ratios are given by equation (18).

The total volume below the critical depth is given by $V_{s}+V_{w}+V_{l o}+V_{g 2}$. Using equations (18), (27), and (34), we obtain the the volume fractions of live oil, water, free gas, and solid:

$$
\begin{aligned}
\phi_{l o} & =\gamma\left[\left(V_{s} / V_{o i}\right)+\left(V_{w} / v V_{w i}\right)+\gamma+\delta\right]^{-1} \\
\phi_{w} & =\frac{1}{v \gamma}\left(V_{w} / V_{w i}\right) \phi_{l o} \\
\phi_{f g} & =\frac{\delta}{\gamma} \phi_{l o}
\end{aligned}
$$

and

$$
\phi_{s}=1-\phi_{l o}-\phi_{w}-\phi_{f g}
$$

respectively.

The saturations are equal to the corresponding fluid volumes divided by the pore volume or equal to the fluid fractions divided by the total porosity $\phi$, which is given by

$$
\phi=\frac{V_{p}}{V_{p}+V_{s}}
$$

where $V_{s}$ is the volume of the solid part. Using equation (21) and the expression for $V_{s}$ obtained above, we obtain

$$
\phi=\frac{\phi_{i} \exp \left[E(\Delta p)+\alpha_{p} \Delta T\right]}{1-\phi_{i}\left\{1-\exp \left[E(\Delta p)+\alpha_{p} \Delta T\right]\right\}} .
$$

Then the free gas, live oil, and water saturations are

$$
S_{f g}=\phi_{f g} / \phi, \quad S_{l o}=\phi_{l o} / \phi, \quad \text { and } \quad S_{w}=\phi_{w} / \phi,
$$

respectively.

Let us calculate the saturations and volume fractions when the gas does not go into solution. As the pore pressure increases from $p_{i}$ to $p$, the pore volume changes from $V_{p i}$ to $V_{p i}\left\{\exp \left[E(\Delta p)+\alpha_{p} \Delta T\right]\right\}$. The saturations are equal to the corresponding volumes divided by the pore volume. Using equations (13) and (21) gives, for the oil and water saturations,

$$
\begin{aligned}
S_{o}= & \frac{v}{1+v}(1-F)\left[\operatorname { e x p } \left(-c_{o} \Delta p+\alpha_{o} \Delta T-E(\Delta p)\right.\right. \\
& \left.\left.-\alpha_{p} \Delta T\right)\right]
\end{aligned}
$$

and

$S_{w}=\frac{1}{1+v}\left[\exp \left(-c_{w} \Delta p+\alpha_{w} \Delta T-E(\Delta p)-\alpha_{p} \Delta T\right)\right]$

respectively. The gas saturation is simply

$$
S_{g}=1-S_{o}-S_{w}
$$

On the other hand, the fluid fractions are

$$
\phi_{o}=\phi S_{o}, \quad \phi_{w}=\phi S_{w}, \quad \text { and } \quad \phi_{g}=\phi S_{g} .
$$




\section{Compressibilities and dry-rock bulk moduli}

The isothermal gas bulk modulus $K_{g}$ and the gas compressibility $c_{g}=K_{g}^{-1}$ depend on pressure. The latter can be calculated from the van der Waals equation as

$$
c_{g}=\frac{1}{\rho_{g}} \frac{\partial \rho_{g}}{\partial p}
$$

at constant temperature. It gives

$$
c_{g}=\left[\frac{\rho_{g} R T}{\left(1-b \rho_{g}\right)^{2}}-2 a \rho_{g}^{2}\right]^{-1} .
$$

The pore compressibility $c_{p}$ is related closely to the bulk modulus of the dry rock $K_{m}$ (the compressibility $c_{p}$ is denoted by $C_{p p}$ in Zimmerman et al. (1986) and by $K_{\phi p}^{-1}$ in Mavko and Mukerji (1995), and $K_{m}$ corresponds to $C_{b c}^{-1}$ and $K_{d r y}^{-1}$, respectively). Using the present notation, $c_{p}$ can approximately be expressed as

$$
c_{p}=\left(\frac{1}{K_{m}}-\frac{1}{K_{s}}\right) \frac{1}{\phi}-\frac{1}{K_{s}},
$$

where $\phi$ depends on the excess pore pressure. Because dryrock wave velocities are practically frequency independent, the seismic bulk moduli $K_{m}$ and $\mu_{m}$ versus confining pressure can be obtained from laboratory measurements in dry samples. If $V_{P}$ and $V_{S}$ are the experimental compressional and shear velocities, the moduli are given approximately by

$$
K_{m}=(1-\phi) \rho_{s}\left(V_{P}^{2}-\frac{4}{3} V_{S}^{2}\right), \quad \mu_{m}=(1-\phi) \rho_{s} V_{S}^{2},
$$

where $\rho_{s}$ is the grain density and $\phi$ is the porosity. We recall that $K_{m}$ is the rock modulus at constant pore pressure, i.e., the case when the bulk modulus of the pore fluid is negligible compared with the dry-rock bulk modulus, as, for example, air at room conditions (Mavko and Mukerji, 1995). Note that gas at high pressures can have a nonnegligible bulk modulus.

\section{Seismic properties of the rock volume}

The seismic velocities of the overpressured porous rock are computed by using Biot's theory of dynamic poroelasticity (Appendix A). The phase velocities of $P$ - and $S$-waves are given by

$$
V_{P \pm}=\left[\operatorname{Re}\left(\frac{1}{V_{P \pm}^{*}}\right)\right]^{-1}, \quad V_{S}=\left[\operatorname{Re}\left(\frac{1}{V_{S}^{*}}\right)\right]^{-1},
$$

where $V_{P \pm}^{*}$ are the complex velocities of the fast $(+)$ and slow $(-)$ waves, $V_{S}^{*}$ is the complex shear wave velocity, and Re takes the real part.

\section{EXAMPLE}

We assume that the medium is saturated fully with oil and that before oil/gas conversion occurs, the initial pressure, $p_{i}$, is hydrostatic. Because there is no water, $v \rightarrow \infty$, and equation (22) becomes

$$
\begin{aligned}
\exp \left[E(\Delta p)+\alpha_{p} \Delta T\right]= & (1-F)\left[\exp \left(-c_{o} \Delta p+\alpha_{o} \Delta T\right)\right] \\
& +F \rho_{o i} / \rho_{g} .
\end{aligned}
$$

When there is dissolved gas, the balance equations are

$$
\exp \left[E(\Delta p)+\alpha_{p} \Delta T\right]=\eta, \quad z \leq z_{c}
$$

and

$$
\begin{aligned}
\exp \left[E(\Delta p)+\alpha_{p} \Delta T\right]= & \gamma+F \rho_{o i} / \rho_{g} \\
& -R_{G}^{\prime}(1-F) \rho_{o i} / \rho_{o}, \quad z>z_{c} .
\end{aligned}
$$

With a surface temperature of $15.6^{\circ} \mathrm{C}$, a temperature gradient $G=25^{\circ} \mathrm{C} / \mathrm{km}$, a sedimentation rate $S=0.08 \mathrm{~km} / \mathrm{m}$.y., and a reservoir volume at $z_{i}=z_{1}=2 \mathrm{~km}$, we have $t_{i}=t_{1}=25 \mathrm{~m}$.y. and $T_{1}=65.6^{\circ} \mathrm{C}$. After 75 m.y., the depth of burial is $z_{2}=8 \mathrm{~km}, t_{2}=100 \mathrm{~m}$.y., and $T_{2}=215.6^{\circ} \mathrm{C}$. On the other hand, if $\bar{\rho}=2.4 \mathrm{~g} / \mathrm{cm}^{3}$, the confining pressure has increased from $47 \mathrm{MPa}$ to approximately $188 \mathrm{MPa}$, and the initial pore pressure is $p_{i} \simeq 20 \mathrm{MPa}$ (assuming $\rho_{w}=1 \mathrm{~g} / \mathrm{cm}^{3}$ ). If no conversion takes place, the final pore pressure would be the hydrostatic pressure at $8 \mathrm{~km}$, i.e., approximately $78 \mathrm{MPa}$.

The experimental data for oil-saturated sandstone are available in Winkler (1985), in his Figures 3 and 4 and Tables 4 and 7. It is important to understand how the data were measured. After a suitable pore vacuum was achieved, confining pressure was increased to $40 \mathrm{MPa}$. Dry-rock wave velocities then were obtained at successive pressures as the confining pressure decreased to $10 \mathrm{MPa}$. While still in the pressure vessel, the sample was saturated with oil to a pore pressure of $5 \mathrm{MPa}$. Differential pressure $p_{c}$ was then increased to 10,20 , and $40 \mathrm{MPa}$, corresponding to the values $\left(p_{c}, p\right)=(20,10),(40,20)$, and $(60,20)$ (in $\mathrm{MPa}$ ). Note that Winkler calls the differential pressure the effective stress. The experiments on dry samples correspond to zero pore pressure. Best-fit plots of the dry-rock compressibility and shear modulus versus confining pressure are

$$
K_{m}^{-1}\left[\mathrm{GPa}^{-1}\right]=0.064+0.122 \exp \left(-p_{c}[\mathrm{MPa}] / 6.48\right)
$$

and

$$
\mu_{m}[\mathrm{GPa}]=13.7-8.5 \exp \left(-p_{c}[\mathrm{MPa}] / 9.14\right),
$$

and $c_{p}$ in $\mathrm{GPa}^{-1}$ is given by equation (16), with $c_{p}^{\infty}=0.155$, $\beta=0.6$, and $p^{*}=6.48$. The pore compressibility $c_{p}$ has been obtained from equation (51) by assuming that the porosity is that at hydrostatic pore pressure [this approximation is supported by experimental data obtained by Domenico (1977) and Han et al. (1986)]. The best-fit plots for $c_{p}$ and $K_{m}^{-1}$ are illustrated in Figure 2.

To obtain the moduli for different combinations of the confining and pore pressures, we should make the substitution $p_{c} \rightarrow p_{e}=p_{c}-n p$, where we assume, following Gangi and Carlson (1996), that $n$ depends on differential pressure as

$$
n=n_{0}-n_{1} p_{d}, \quad n_{0}=1, \quad n_{1}=0.014 \mathrm{MPa}^{-1} .
$$

This dependence of $n$ versus differential pressure is in good agreement with the experimental values corresponding to the compressional velocity obtained by Christensen and Wang (1985) and by Prasad and Manghnani (1997).

Table 1 indicates the properties for Berea sandstone, where the values correspond to those at the initial (hydrostatic) pore pressure. The oil and gas viscosities as a function of temperature and pore pressure are taken from Luo and Vasseur (1996).

The procedure for computing the phase velocities and attenuation factors is the following:

1) Compute the oil/gas conversion factor as a function of deposition time and depth by using equations (4) and (10). 
2) Compute the dry-rock bulk moduli (52) from the experimental wave velocities $V_{P}\left(p_{c}\right)$ and $V_{S}\left(p_{c}\right)$. This also gives the dry-rock bulk moduli as a function of effective pressure. We assume that the porosity is that at hydrostatic pressure.

3) Compute the initial gas density and the gas density at pressure $p, \rho_{g}(p)$ from the van der Waals equation (11) and the pore compressibility from equation (51). As before, we assume that the porosity is that at hydrostatic pressure.

4) Compute the excess pore pressure corresponding to the oil/gas conversion factor $F$ by using equations (54) or (55) and (56), and the oil and gas saturations by using equations (45) or (46) and (48). Overpressure occurs when pore pressure exceeds hydrostatic pressure.

5) The low-frequency wet-rock velocities are computed by using the porous model (Appendix A), and the dilatational and shear relaxation times are obtained by fitting the experimental high-frequency wet-rock velocities at full saturation, i.e., at the onset of the oil/gas conversion.

6) Phase velocities and attenuation factors are computed as

Table 1. Material properties for Berea sandstone.

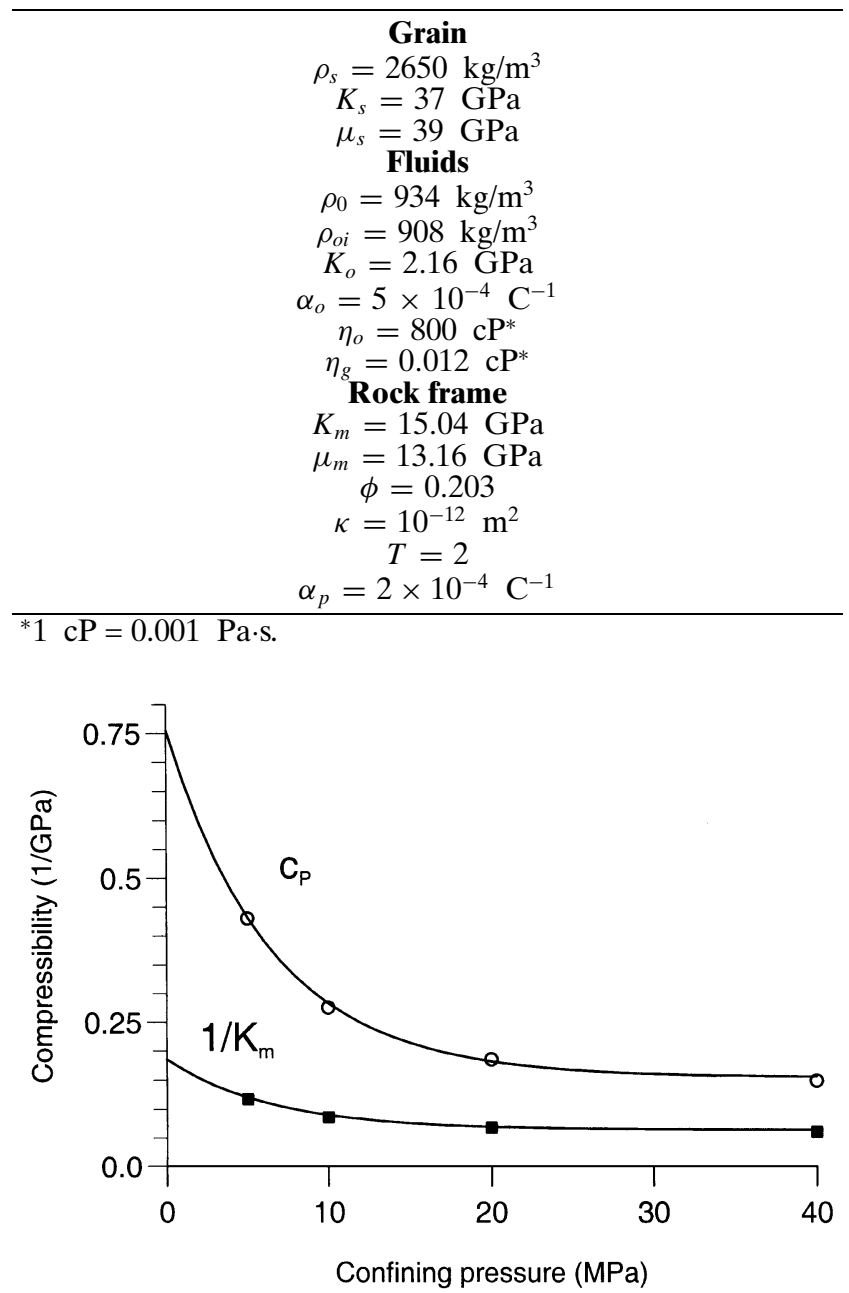

FIG. 2. Best-fit plots of $c_{p}$ and $K_{m}^{-1}$ obtained from the experimental data for Berea sandstone published by Winkler (1985, Figures 3 and 4 and Tables 4 and 7). a function of pore pressure and frequency from equations (A-15) and (A-16), respectively.

Figure 3 shows the oil/gas conversion factor $F$ [equation (4)] (a) and its logarithm (b) as a function of depth and time. The high activation energy requires either a long time or deep burial, on the order of 4.5 to $5 \mathrm{~km}$, before appreciable fractions of conversion occur, but significant fractional conversions occur at $3 \mathrm{~km}$. The pore-pressure buildup with depth is
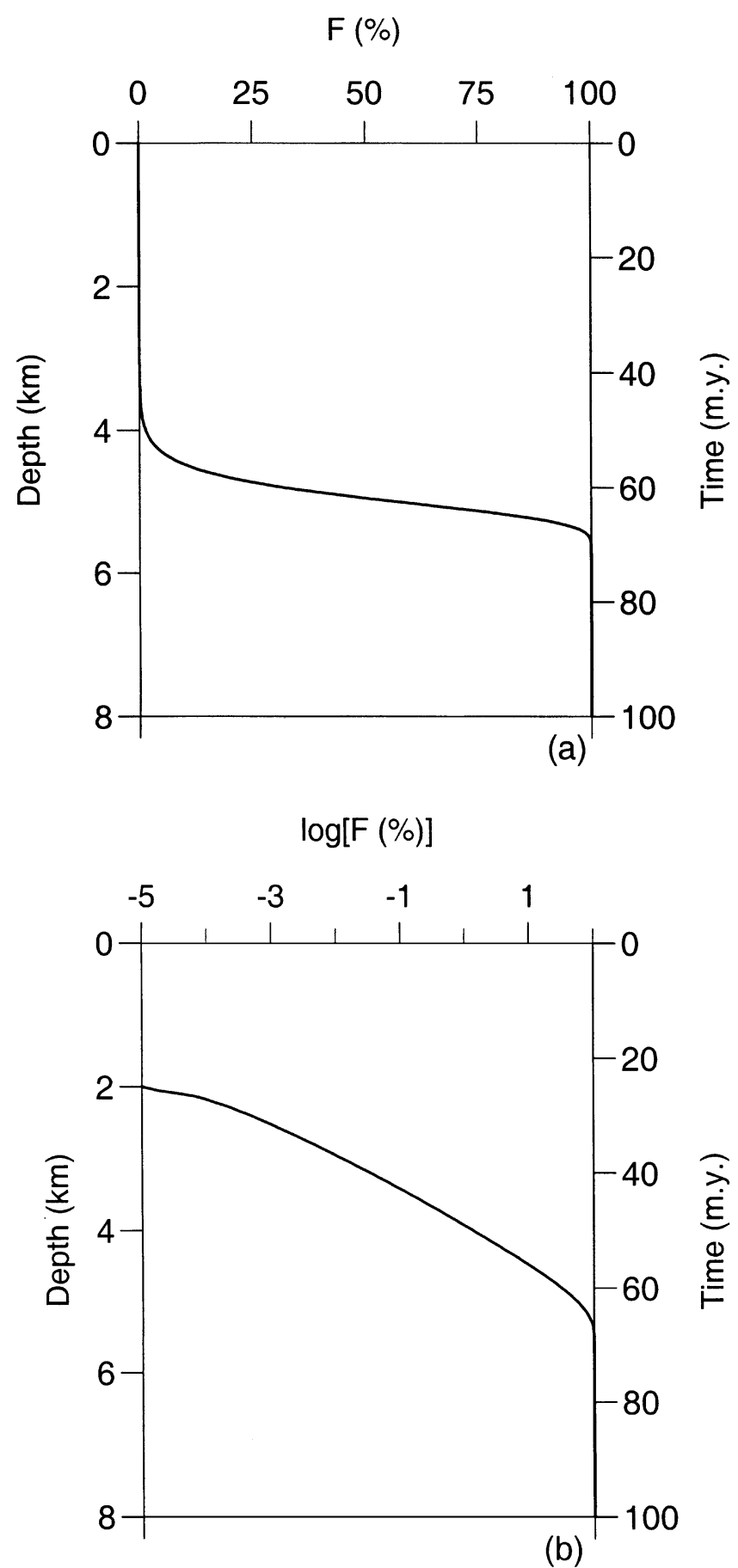

FIG. 3. Oil/gas conversion factor $F$ [equation (4)] (a) and $\log (F)(\mathrm{b})$ as a function of depth and time. 
shown in Figure 4, for all the generated gas out of solution (continuous line), and for part of the generated gas dissolved in the oil (broken line). The two cases yield practically the same result. The pressure increases rapidly for very small fractions of oil converted to gas. This is caused by the expansion of the pore volume resulting from generation of free gas or live oil. Beyond the lithostatic pressure, the effective pressure becomes negative and the pore compressibility increases (see Figure 2), making the rock highly compliant. This precludes a rapid increase of the pore pressure, which follows the lithostatic pressure below $4 \mathrm{~km}$ depth. The pore pressure equals the lithostatic pressure when $2.5 \%$ of the oil has been converted to gas, which occurs at a depth of approximately $4.2 \mathrm{~km}$. In the case of dissolved gas, the pore pressure equals the lithostatic pressure without generation of free gas. It is caused by the increase in live-oil volume, since the critical depth is $z_{c}=5.05 \mathrm{~km}$ and $F_{c}=20 \%$. If the hydrostatic pressure is used as reference profile to solve equations (33) and (35), we obtain $z_{c}=4.77 \mathrm{~km}$ and $F_{c}=7 \%$ and practically the same pore-pressure buildup shown in Figure 4. Because the results with and without dissolved gas are practically the same, we consider in the following analysis the case with no dissolved gas. Figure 5 shows the variations of the saturations with excess pressure (a) and of the porosities with depth (b) [(equations (46), (48), and (49), respectively]. The arrows indicate when the pore pressure equals the lithostatic pressure.

The low-frequency wave velocities versus excess pore pressure and differential pressure are shown in Figure 6. An excess pore pressure of $60 \mathrm{MPa}$ corresponds to zero differential pressure. The oil/gas conversion starts at a differential pressure of 21.5 $\mathrm{MPa}$, which corresponds to an onset time of approximately

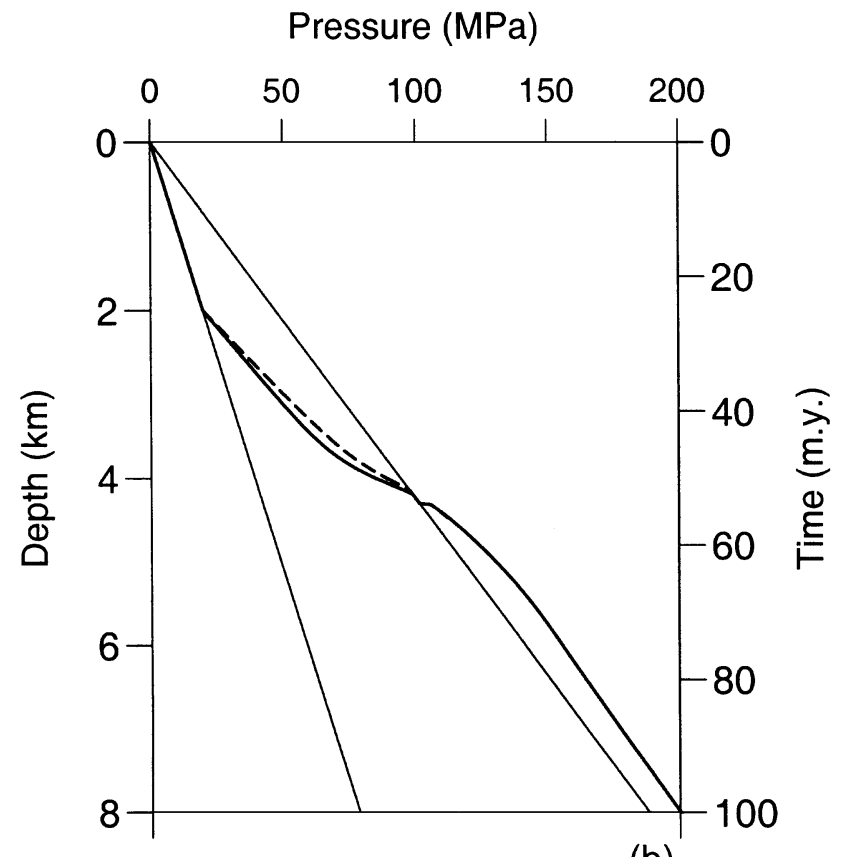

(b)

FIG. 4. Pore-pressure buildup with depth and deposition time (continuous line), when all the generated gas is out of solution (continuous line), and when part of the generated gas is dissolved in the oil (broken line). The hydrostatic and lithostatic pressure are represented by thin lines.
25 million years, when the sandstone is fully saturated with oil. As can be appreciated, the velocities decrease substantially after an excess pore pressure of approximately $35 \mathrm{MPa}$. This results partly from the replacement of oil by gas, but mainly from the decrease in the dry-rock bulk moduli $K_{m}$ and $\mu_{m}$ caused by the decrease in effective pressure.

Winkler (1985) obtained the high-frequency compressional and shear velocities, $4140 \mathrm{~m} / \mathrm{s}$ and $2500 \mathrm{~m} / \mathrm{s}$, at a differential pressure of $27.4 \mathrm{MPa}$. These experimental velocities, corresponding to $400 \mathrm{kHz}$, are fitted with the theoretical velocities (A-15) to obtain the relaxation times used in equations (A-10) and (A-11). The pores are saturated with dead oil of density $890 \mathrm{~kg} / \mathrm{m}^{3}$, which corresponds to the value reported by Winkler (1985). Figure 7 shows the normalized compressional (continuous lines) and shear (broken lines) velocities (a) and attenuations (b) versus frequency. The velocities are normalized with respect to the low-frequency values $4013 \mathrm{~m} / \mathrm{s}$ and $2426 \mathrm{~m} / \mathrm{s}$, respectively. The square and circle correspond
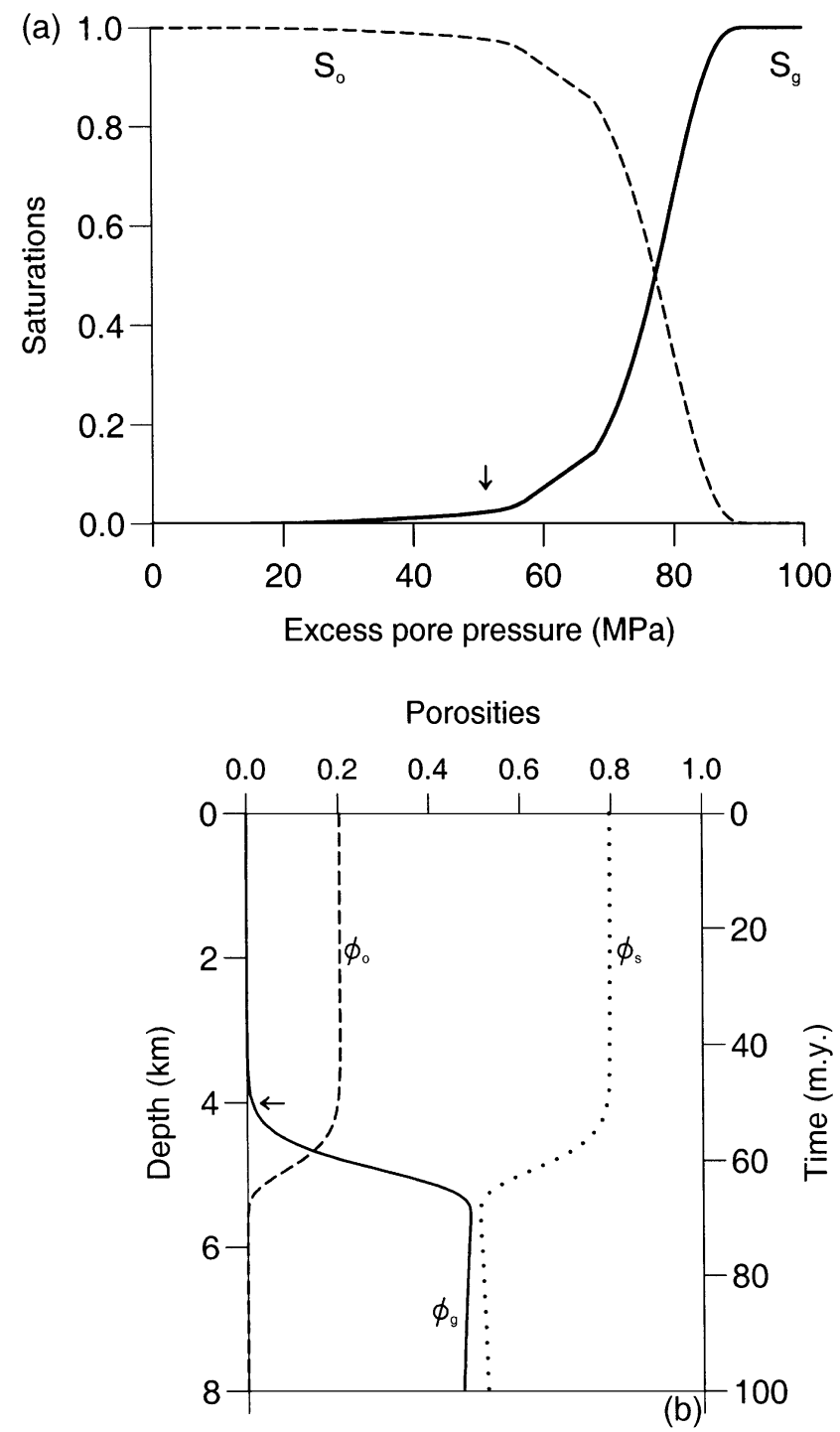

FIG. 5. Saturations versus excess pore pressure $p-p_{H}$ (a) and porosities versus depth (b). The arrows indicate when the pore pressure equals the lithostatic pressure. 
to the experimental values obtained by Winkler (1985) for the compressional and shear waves, and the triangle is the $P$-wave attenuation for a differential pressure of $40 \mathrm{MPa}$ [attenuation $\alpha$, in $\mathrm{dB}$, is related to $Q$ factor as $\alpha \simeq 17.372 \pi /(2 Q)]$. To fit these values, we use one mechanism for the shear modulus $\mu_{c}$, centered at $4 \mathrm{kHz}$ and with relaxation times $\tau_{\epsilon}=4.09 \times 10^{-5} \mathrm{~s}$ and $\tau_{\sigma}=3.86 \times 10^{-5} \mathrm{~s}$ (corresponding to a mimimum quality factor of approximately 34), and a continuous spectrum of mechanisms for $M_{c}$ from $1 \mathrm{~Hz}$ to $1 \mathrm{MHz}$ and $\bar{Q} \simeq 25$. The need for a continuous spectrum of relaxation mechanisms to explain the $P$-wave attenuation values is demonstrated in Winkler (1985), but because of lack of data for the shear wave, we assume the simplest model, a single relaxation mechanism.

The Biot relaxation mechanisms, resulting from the relative motion between the solid and the fluids, are important beyond $1 \mathrm{MHz}$. The peaks at $4 \mathrm{KHz}$ result mainly from the shear viscoelastic mechanism.
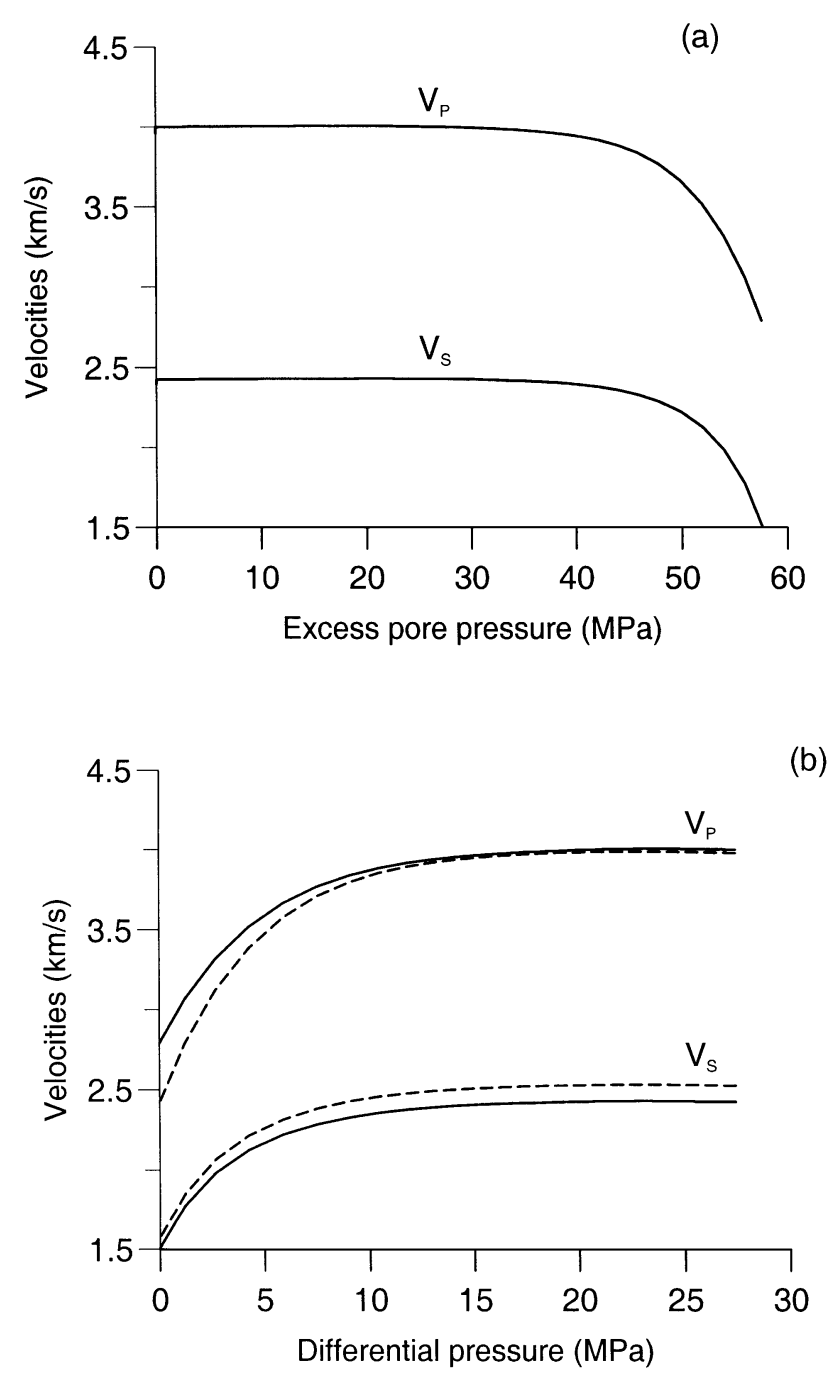

FIG. 6. Computed low-frequency wave velocities versus excess pore pressure (a) and differential pressure (b). Frequency is $25 \mathrm{~Hz}$. The broken lines in (b) are the dry-rock wave velocities derived from equations (57) and (58).

\section{CONCLUSIONS}

The variations of $P$-wave and $S$-wave velocities have been determined as a function of excess pressure caused by oil/gas conversion. The results for a model in which a reservoir volume is buried at a constant sedimentation rate for a geothermal gradient, which is constant in time and depth, show that the velocities decrease significantly when only a small amount (about $2.5 \%$ ) of the oil in the closed reservoir is converted to gas. If the gas goes into solution, the results are practically the same, because the volume increase of the live oil is close to that of the mixture of dead oil/free gas. Moreover, the differential pressure vanishes before the oil has absorbed all the possible generated gas.
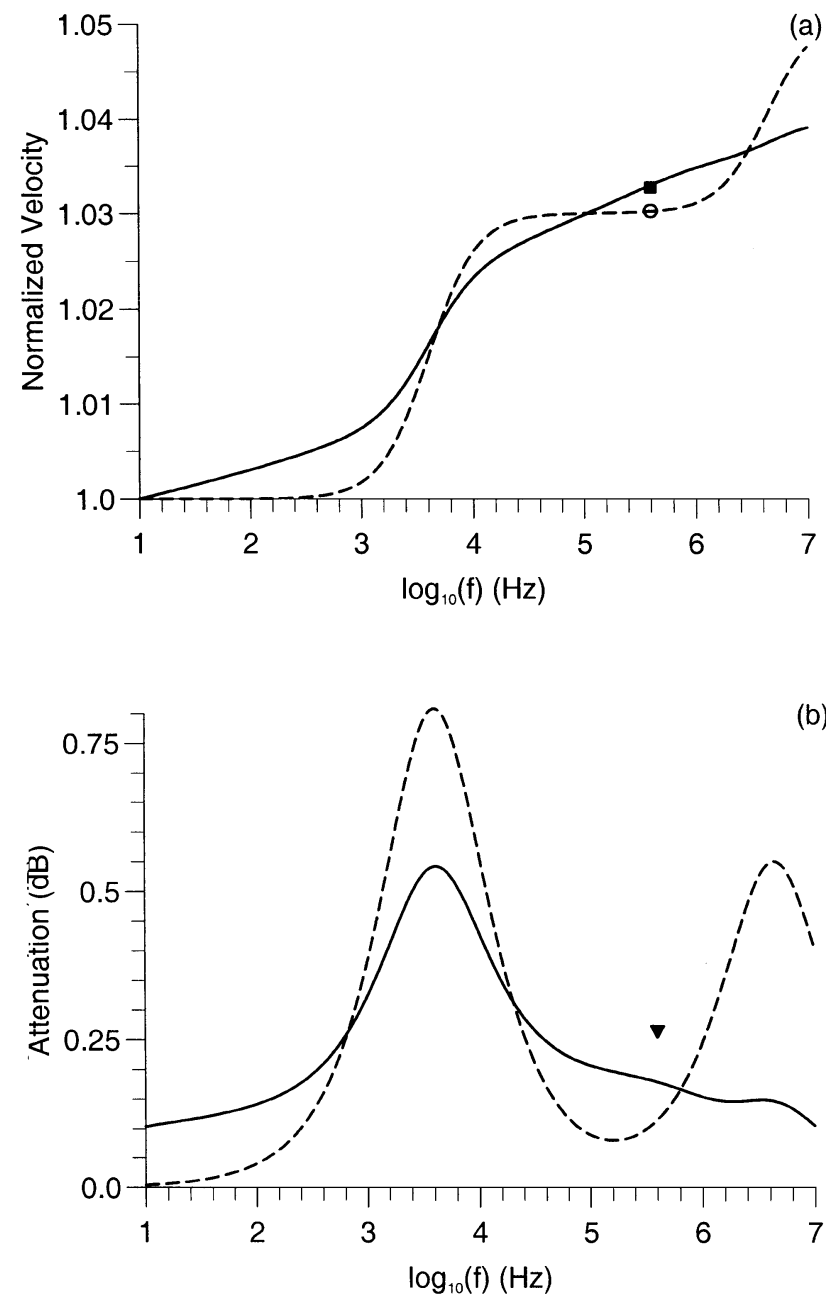

FIG. 7. Normalized compressional (continuous line) and shear (broken line) velocities (a) and attenuations (b) versus frequency, for a differential pressure of 27.4 MPa. The velocities are normalized with respect to the low-frequency values $4013 \mathrm{~m} / \mathrm{s}$ and $2426 \mathrm{~m} / \mathrm{s}$, respectively. They correspond to the onset of the oil/gas conversion process. The square and circle at $400 \mathrm{kHz}$ correspond to the experimental values obtained by Winkler (1985) for the compressional and shear waves, and the triangle at $400 \mathrm{kHz}$ is the $P$-wave attenuation for a differential pressure of $40 \mathrm{MPa}$. 
That small conversion of oil to gas is sufficient to make the pore pressure equal to the confining pressure. The large change in the velocity results mainly from the fact that the dry-rock moduli are functions of the effective pressure, with the largest changes occurring at low effective pressures. In fact, porosity changes and fluid moduli and density have a second-order effect on the velocities. The effective pressure decreases because the pore pressure increases as a result of conversion of highdensity oil to the low-density gas or low-density live oil, in the case of dissolved gas. Perceptible changes in velocities occur when the differential pressure is $15 \mathrm{MPa}$, at which about $0.6 \%$ of oil has been converted to gas. The changes become significant when the differential pressure decreases to $10 \mathrm{MPa}$, at which about $1 \%$ has been converted.

Attenuation also will be a function of effective pressure because of the changing pore-space geometry. However, experimental data of attenuation for partially saturated rocks as a function of the effective pressure are not available. Realistic attenuation values were obtained only for the case with full oil saturation, for which experimental evidence of the velocity dispersion is available.

\section{ACKNOWLEDGMENTS}

This work was supported by Norsk Hydro a.s. (Bergen) with funds of the "Source Rock" project, and by the European Union, under the project "Detection of overpressure zones with seismic and well data." Thanks go to Hans Helle for important technical comments.

\section{REFERENCES}

Barker, C., 1990, Calculated volume and pressure changes during the thermal cracking of oil to gas in reservoirs: AAPG Bull., 74, 12541261.

Batzle, M., and Wang, Z., 1992, Seismic properties of pore fluids: Geophysics, 57, 1396-1408.

Berryman, J. G., Thigpen, L., and Chin, R. C. Y., 1988, Bulk elastic wave propagation in partially saturated porous solids: J. Acoust. Soc. Am., 84, no. 1, 360-373.

Berg, R. R., and Gangi, A. F., 1999, Primary migration by oil-generation microfracturing in low-permeability source rocks: Application to the Austin chalk, Texas: AAPG Bull., 83, no. 5, 727-756.

Carcione, J. M., 1998, Viscoelastic effective rheologies for modeling wave propagation in porous media: Geophys. Prosp., 46, 249-270.
2000, A model for seismic velocity and attenuation in petroleum source rocks: Geophysics, 65, 1080-1092.

Chaney, P. E., 1950, Abnormal pressure and lost circulation: World Oil, 130, 122-126.

Christensen, N. I., and Wang, H. F., 1985, The influence of pore pressure and confining pressure on dynamic elastic properties of Berea sandstone: Geophysics, 50, 207-213.

Dasios, A., Astin, T. R., and McCann, C., 1998a, Compressional-wave attenuation from full waveform sonic data: 60th Ann. Internat. Mtg., Europ. Assoc. Geophys. Eng., Expanded Abstracts, 10-44.

1998b, Increasing confidence in seismic Q measurements: A comparison of estimates from sonic and surface seismic data: 68th Ann. Internat. Mtg., Soc. Expl. Geophys., Expanded Abstracts, 1080-1083.

Domenico, S. N., 1977, Elastic properties of unconsolidated porous sand reservoirs: Geophysics, 42, 1339-1368.

Dutta, N. C., and Levin, F. K., 1990, Geopressure, Geophysical Reprint Series No. 7, Soc. Expl. Geophys.

Dvorkin, J., Nolen-Hoeksema, R., and Nur, A., 1994, The squirt-flow mechanism: Macroscopic description: Geophysics, 59, 428-438.

Friedman, A. S., 1963, Pressure-volume-temperature relationships of gases, virial coefficients, in American Institute of Physics handbook: McGraw-Hill Book Co.

Gangi, A. F., and Carlson, R. L., 1996, An asperity-deformation model for effective pressure: Tectonophysics, 256, 241-251.

Gautschi, W., and Cahill, W. F., 1964, Exponential integral and related functions, in M. Abramowitz and I. A. Stegun, Eds., Handbook of mathematical functions with formulas, graphs and mathematical tables: U. S. Department of Commerce, National Bureau of Standards, Applied Math. Series 55, S5, 227-254.

Hague, P. S., 1981, Measurements of attenuation from vertical seismic profiles: Geophysics, 46, 1548-1558.

Han, D., Nur, A., and Morgan, D., 1986, Effects of porosity and clay content on wave velocities in sandstones: Geophysics, 51, 2093-2107.

Helle, H. B., Inderhaug, O. H., Kovaliev, V. P., Madatov, A., and Mitrofanov, G. M., 1993, Complex seismic decompositionApplication to pore pressure prediction, 55th Ann. Internat. Mtg., Europ. Assoc. Expl. Geophys., Expanded Abstracts, B005.

Luo, X., and Vasseur, G., 1996, Geopressuring mechanism of organic matter cracking: Numerical modeling: AAPG Bull., 80, 856874.

Mann, D. M., and Mackenzie, A. S., 1990, Prediction of pore fluid pressures in sedimentary basins: Marine and Petroleum Geology, 7, $55-65$.

Mavko, G., and Mukerji, T., 1995, Seismic pore space compressibility and Gassmann's relation: Geophysics, 60, 1743-1749.

Prasad, M., and Manghnani, M. H., 1997, Effects of pore and differential pressure on compressional wave velocity and quality factor in Berea and Michigan sandstones: Geophysics, 62, 11631176.

Standing, M. B., 1952, Volumetric and phase behavior of oil field hydrocarbon systems: Reinhold.

Winkler, K. W., 1985, Dispersion analysis of velocity and attenuation in Berea sandstone: J. Geophys. Res., 90, 6793-6800.

Zimmerman, R. W., Somerton, W. H., and King, M. S., 1986, Compressibility of porous rocks: J. Geophys. Res., 91, 12765-12777.

\section{APPENDIX A}

\section{SEISMIC PROPERTIES OF A POROUS MEDIUM SATURATED WITH HYDROCARBON AND WATER}

Biot's theory of dynamic poroelasticity is used to compute the wave velocities and attenuation factors, where the pore fluid is a mixture of hydrocarbon and water. The complex velocities of the fast $(+$ sign $)$ and slow $(-$ sign $)$ compressional waves and shear wave are [see Carcione (1998)],

$$
V_{P \pm}^{* 2}=\frac{A \pm \sqrt{A^{2}-4 M E \rho_{c} \rho^{*}}}{2 \rho_{c} \rho^{*}}
$$

and

$$
V_{S}^{* 2}=\frac{\mu}{\rho_{c}}
$$

where

$$
A=M\left(\rho-2 \alpha \rho_{f}\right)+\rho^{*}\left(E+\alpha^{2} M\right)
$$

$$
\rho_{c}=\rho-\rho_{f}^{2} / \rho^{*}
$$

and

$$
\rho^{*}=\frac{\mathcal{T}}{\phi} \rho_{f}-\frac{i}{2 \pi f} \frac{\eta}{\kappa},
$$

with $\alpha$ and $E$ elastic coefficients, $f$ the frequency, and $i=\sqrt{-1}$. The sediment density is

$$
\rho=(1-\phi) \rho_{s}+\phi \rho_{f}
$$

where $\rho_{s}$ and $\rho_{f}$ are the solid and fluid densities, respectively; $\mathcal{T}$ is the tortuosity; $\eta$ is the fluid viscosity; and $\kappa$ is the permeability of the medium. 
The elastic coefficients are given by

$$
\begin{aligned}
E & =K_{m}+\frac{4}{3} \mu, \\
M & =\frac{K_{s}^{2}}{D-K_{m}}, \\
D & =K_{s}\left[1+\phi\left(K_{s} K_{f}^{-1}-1\right)\right], \\
\alpha & =1-\frac{K_{m}}{K_{s}},
\end{aligned}
$$

with $K_{f}$ the bulk modulus of the mixture hydrocarbon/water. The stiffness $E$ is the $P$-wave modulus of the dry skeleton, $M$ is the elastic coupling modulus between the solid and the fluid, and $\alpha$ is the poroelastic coefficient of effective stress.

Wave velocities generally are expected to be lower at low frequencies, typical of seismic measurements, than at high frequencies, typical of laboratory experiments. Because the magnitude of this effect cannot be described entirely by Biottype theories, additional relaxation mechanisms are required to model the velocity dispersion. Measurements of dry-rock velocities contain all the information about pore shapes and pore interactions and their influence on wave propagation. Low-frequency wet-rock velocities can be calculated by using Gassmann's equation, i.e., the low-frequency limit of the dispersion relation. High-frequency wet-rock velocities are then given by the unrelaxed velocities. Because dry-rock velocities are practically frequency independent, the data can be obtained from laboratory measurements

Viscoelasticity is introduced into the poroelastic equations for modeling a variety of dissipation mechanisms related to the skeleton/fluid interaction. One of these mechanism is the squirt-flow (Dvorkin et al., 1994), by which a force applied to the area of contact between two grains produces a displacement of the surrounding fluid in and out of this area. Because the fluid is viscous, the motion is not instantaneous and energy dissipation occurs. Skeleton/fluid mechanisms are modeled by generalizing $M$ to a frequency-dependent modulus of the form

$$
M_{c}=M\left[1+\frac{2}{\pi \bar{Q}} \ln \left(\frac{1+i \omega \tau_{2}}{1+i \omega \tau_{1}}\right)\right]^{-1}
$$

where $\tau_{1}$ and $\tau_{2}$ are time constants, with $\tau_{2}<\tau_{1}$, and $\bar{Q}$ defines the value of the quality factor, which remains nearly constant over the selected frequency band. The low-frequency limit of $M_{c}$ is the Biot modulus $M$.

On the other hand, $\mu$ is generalized to

$$
\mu_{c}=\frac{\mu}{L} \sum_{l=1}^{L} \frac{1+i \omega \tau_{\epsilon l}}{1+i \omega \tau_{\sigma l}},
$$

where $\tau_{\epsilon l}$ and $\tau_{\sigma l}$ are the relaxation times of the $L$ attenuation mechanisms.

The mixture hydrocarbon/water behaves as a composite fluid with properties depending on the constants of the constituents and their relative concentrations. This problem has been analyzed by Berryman el al. (1988), and the results are given by the formulas

$$
\begin{gathered}
K_{f}=\left(S_{o} c_{o}+S_{w} c_{w}\right)^{-1}, \\
\rho_{f}=S_{o} \rho_{o}+S_{w} \rho_{w},
\end{gathered}
$$

where $\rho_{o}$ is the density of the hydrocarbon,

$$
\eta_{f}=S_{o} \eta_{o}+S_{w} \eta_{w},
$$

where $\eta_{o}$ and $\eta_{w}$ are the viscosities of the hydrocarbon and water, respectively. Equation (A-14) is a good approximation for most values of the saturations.

The phase velocity $V_{P(S)}$ is equal to the angular frequency $\omega=2 \pi f$ divided by the real wavenumber. Then

$$
V_{P \pm}=\left[\operatorname{Re}\left(\frac{1}{V_{P \pm}^{*}}\right)\right]^{-1}, \quad V_{S}=\left[\operatorname{Re}\left(\frac{1}{V_{S}^{*}}\right)\right]^{-1}
$$

where Re takes the real part.

The magnitude of the attenuation factors (in $\mathrm{dB}$ ) is given by

$$
\alpha_{P \pm}=17.372 \pi \frac{\operatorname{Im}\left(V_{P \pm}^{*}\right)}{\operatorname{Re}\left(V_{P \pm}^{*}\right)}, \quad \alpha_{S}=17.372 \pi \frac{\operatorname{Im}\left(V_{S}^{*}\right)}{\operatorname{Re}\left(V_{S}^{*}\right)},
$$

where Im takes the imaginary part.

Biot's theory is strictly valid for frequencies below

$$
f_{c}=\frac{\eta_{f} \phi}{2 \pi \mathcal{T} \rho_{f} \kappa} .
$$

